\title{
Veränderungen in der Aktivität Chondroitinsulfat-Protein abbauender Enzyme (Glykosaminoglykanohydrolasen und Peptidhydrolasen) des Arteriengewebes im Alter und bei Arteriosklerose
}

\author{
Von H. Kresse und E. Buddecke \\ Aus dem Pbysiologisch-Chemischen Institut der Universität Münster
}

(Eingegangen am 5. Dezember 1967)

Herrn Prof. Dr. Dr. Ernst Schütte zum 60. Geburtstag genidmet

Im Arteriengewebe von Rind und Mensch (Aorta thoracica) wurden Veränderungen der Aktivität der Glucosidasen N-Acetyl- $\beta$-D-glucosaminidase (EC 3.2.1.30), $\beta$-D-Glucuronidase (EC 3.2.1.31) und Hyaluronat-Glucanohydrolase (EC 3.2.1.35) sowie der Peptidhydrolasen Kathepsin D (EC 3.4.4.23) und saure Carboxypeptidase (EC 3.4.2....) in Abhängigkeit vom Lebensalter und Arteriosklerose bestimmt. Die ermittelten Enzymaktivitäten werden auf den DNA-Gehalt des Gewebes bezogen.

In der Aorta des Rindes zeigen die Aktivitäten von $\mathrm{N}$-Acetyl- $\beta$-D-glucosaminidase und $\beta$-D-Glucuronidase keine gerichteten altersabhängigen Vetänderungen, obgleich der Gehalt an Desoxyribonucleinsäure während des ersten Lebensjahres um $50 \%$ abfällt. Die Aktivität der Hyaluronidase sinkt innerhalb von 13 Lebensjahren um mehr als die Hälfte ab, Kathepsin $\mathrm{D}$ und saure Carboxypeptidase zeigen dagegen innerhalb von 7 Jahren einen kontinuierlichen Anstieg ihrer Aktivität bis auf mehr als $100 \%$.

Bei Untersuchung von 85 Gewebsproben menschlicher Arterien wurde ein Anstieg der Enzymaktivität für die Enzyme N-Acetyl- $\beta$-D-glucosaminidase, $\beta$-D-Glucuronidase, Kathepsin D und die saure Carboxypeptidase mit zunehmendem Lebensalter und zunehmender Arteriosklerose beobachtet. Trotz starker Streuung der Einzelwerte läßt sich der Anstieg, der bei einem Vergleich der Mittelwerte der Enzymaktivitäten bei Neugeborenen und einer Altersgruppe von $60-80$ Jahren mehr als $100 \%$ beträgt, statistisch sichern. Im gleichen Zeitraum nimmt der Gehalt des Arteriengewebes an Desoxyribonucleinsäure um mehr als $50 \%$ ab. Bei der $\beta$-Glucuronidase und Protease weisen ferner Gewebsproben mit geringgradigen arteriosklerotischen Veränderungen (Frühstadium) eine statistisch signifikante höhere spezifische Aktivität auf als Gewebe mit fortgeschrittenen Arterioskleroseprozessen.

Die Ergebnisse zeigen, daß die Spontanarteriosklerose des Menschen mit einem Aktivitätsanstieg der katabolen Enzyme des Mucopolysaccharidstoffwechsels einhergeht.

The effect of age and atherosclerosis on the activities of the glucosidases, $\mathrm{N}$-acetyl- $\beta$ - $\mathrm{D}$-glucosaminidase (EC 3.2.1.30), $\beta$ - $\mathrm{D}$-glucuronidase (EC 3.2.1.31) and hyaluronate-glucanohydrolase (EC 3.2.1.35), and the peptide hydrolases, cathepsin D (EC 3.4.4.23) and acid carboxypeptidase (EC 3.4.2. ...) in bovine and human arterial tissue (Aorta thoracica) was studied. The enzyme activities are expressed on the basis of the DNA content of the tissue.

In bovine aorta, the activities of $\mathrm{N}$-acetyl- $\beta$-D-glucosaminidase and $\beta$-D-glucuronidase show no clear correlation with age, although the level of deoxyribonucleic acid falls by $50 \%$ during the first year after birth. During the first 13 years the activity of hyaluronidase falls by more than a half, while cathepsin $\mathrm{D}$ and acid carboxypeptidase undergo a continuous increase in activity by more than $100 \%$ within the first 7 years.

Investigation of 85 tissue samples of human aorta showed that $\mathrm{N}$-acetyl- $\beta$-D-glucosaminidase, $\beta$-D-glucuronidase, cathepsin $\mathrm{D}$ and acid carboxypeptidase increase with increasing age and increasing atherosclerosis. Despite a wide scatter of the individual values, which give a $100 \%$ difference between the averages for newly born and the $60-80$ age group, the increase is statistically significant. Over the same time period the level of deoxyribonucleic acid falls by more than $50 \%$ in the arterial tissue. In tissue samples showing only early stages of atherosclerotic changes, the $\beta$-glucuronidase and protease are significantly higher than in tissue in an advanced stage of atherosclerosis.

'The results show that spontaneous atherosclerosis in humans is correlated with an increase in the activity of the catabolic enzymes of mucopolysaccharide metabolism.

Im katabolen Stoffwechsel des Arteriengewebes werden - Chondroitinsulfat bzw. seine makromolekularen Proteinverbindungen durch Chondroitinsulfat abbauende Enzyme (Glykosaminoglykanohydrolasen) und Protein spaltende Enzyme (Peptidhydrolasen) abgebaut (1). Untersuchungen über altersabhängige Veränderungen in der Aktivität der Glykosaminoglykanohydrolasen sind aus 2 Gründen von Interesse: Einmal ändert sich das Verteilungsmuster der sauren Glykosaminoglykane im menschlichen Arteriengewebe in Abhängigkeit vom Lebensalter und von der Arteriosklerose (2), zum anderen lassen Untersuchungen bei tierexperimenteller Arteriosklerose und bei menschlicher Spontanarteriosklerose, bei denen ein erhöhter Einbau von 35-Sulfat in die sulfatierten Polysaccharide nachgewiesen wurde (3), vermuten, daß Zusammenhänge zwischen der Aktivität von Enzymen des Mucopolysaccharidstoffwechsels und der Genese der Arteriosklerose bestehen. In der vorliegenden Arbeit werden Ergebnisse über altersabhängige Aktivitätsveränderungen der Glykosaminoglykanohydrolasen und Peptidhydrolasen im Arteriengewebe des Rindes (Kapitel 2) und Veränderungen dieser Enzymaktivitäten in menschlichen Arterien in Abhängigkeit vom Alter und Arteriosklerose (Kapitel 3) mitgeteilt.

\section{Methodik \\ Untersucbungsgut}

Für die Untersuchungen zur Änderung der Enzymaktivität im Arteriengewebe mit zunehmendem Lebensalter wurden topographisch identische Proben (Aorta thoracica) von fetalen und neugeborenen Kälbern sowie Rindern verschiedener Altersklassen gesammelt und sofort oder nach Aufbewahrung bei $-20^{\circ}$, wie unten beschrieben, weiter verarbeitet.

Von menschlichen Aorten verschiedenen Lebensalters und beiderlei Geschlechts wurde während der Sektion ein etwa $4 \mathrm{~cm}$ langer Gefäßabschnitt (Abgang der 4.-6. Intercostalarterie) entnommen (Sektionsgut des Pathologischen Instituts der Universität Münster) und nach sorgfältiger Präparation, gegebenenfalls Trennung in Gewebeanteile mit geringerem und stärkerem 
arteriosklerotischen Befall und Ermittlung des Frischgewichtes bei $-20^{\circ}$ bis zur Weiterverarbeitung aufbewahrt. Insgesamt wurden 85 Gewebsproben untersucht.

\section{Enzumaktivitätsbestimmung}

Zur Enzymaktivitätsmessung wurde 1,0-4,0 g Arterien-Frischgewebe nach Vorzerkleinerung in $10-30 \mathrm{~m} / 1$ proz. NaCl-Lösung oder 0,15M Citratpuffer $\mathrm{pH} 4,11 \mathrm{Min}$. unter Kühlung bei $-10^{\circ}$ homogenisiert (Ultraturrax) und vom Uberstand des Homogenats, der durch Zentrifugieren bei $78000 \mathrm{~g}$ gewonnen wurde, $0,1-0,3 \mathrm{~m} /$ zur Enzymaktivitätsbestimmung eingesetzt. Um bei Aktivitätsmessungen der Hyaluronat-Glykanohydrolase den Fehler durch die gleichzeitige Anwesenheit und Wirkung von $\beta$-D-glucuronidase und N-Acetyl- $\beta$-D-glucosaminidase auszuschalten, wurde das Homogenat nach 1. c. (4) einer Ammoniumsulfatfraktionierung und Gelfiltration unterworfen. Die in spezifischen Einheiten (mU/ $\mu$ g-Atom DNA-Phosphor) angegebenen Enzymaktivitäten wurden nach vorstehend (1) mitgeteilten Methoden bestimmt.

\section{Analyse}

Die Bestimmung des Gebaltes an Desoxyribonucleinșäure (DNA) in Arteriengewebsproben wurde im Homogenat bzw. (mit gleichem Ergebnis) im unlöslichen Rückstand des Homogenats nach Ultrazentrifugieren nach 1. c. (5) durchgeführt. Die Proteinbestimmung erfolgt mit Hilfe der Biuretreaktion bzw. - wenn der Proteingehalt des Homogenats weniger als $1 \mathrm{mg} / \mathrm{ml}$ betrug durch Anfärbung der auf Elektrophoresepapier aufgetragenen und getrockneten Probe mit Amidoschwarz $10 \mathrm{~B}$ mit nachfolgender Elution des Farbstoffes nach 1. c. (6).

\section{Ergebnisse}

1. Bezugssystem bei Enzym-Aktivitätsbestimmungen im Arteriengewebe

Unter den möglichen Bezugsgrößen für die Angaben über Enzymaktivitäten (Frischgewicht, Trockengewicht, Protein- oder DNA-Gehalt) ist der Gehalt an DNA im Arteriengewebe aus folgenden Gründen vorzuziehen: Bei Bezug auf das Frisch- oder Trockengewicht gehen die altersabhängigen und Arterioskleroseabhängigen Änderungen im Wasser- und Lipidgehalt des Arteriengewebes als unbekannte Größen ein. Der hierdurch entstehende Fehler kann erheblich sein, da die Lipidkonzentration bei schweren Arteriosklerosen bis zu 30\% des Trockengewichtes (normal 5\%) zunehmen, der Wassergehalt dagegen bis zu $15 \%$ abnehmen kann. Die Angabe von spezifischen Aktivitäten ( $\mu$ Mol-umgesetztes Substrat/Min./mg Protein) ist dadurch mit erheblichen Fehlerquellen belastet, daß einerseits die extrazellulären unlöslichen Strukturproteine Kollagen und Elastin in großem Überschuß vorhanden sind und der Gehalt an löslichen Proteinen im Arteriengewebe (Überstand des Homogenates) altersabhängig ansteigt. Eine die effektive Stoffwechselaktivität des Arteriengewebes widerspiegelnde $\mathrm{Be}$ zugsgröße stellt der Gehalt an DNA dar, da 1. alle somatischen Zellen eines Organismus einen konstanten Gehalt an DNA/Zelle aufweisen, da 2. jedes Enzym zellulären Ursprungs ist und 3. Enzymaktivität und Stoffwechselkapazität der Zelle in direkter Beziehung stehen. Die Bestimmung des DNA-Gehaltes ergibt die Möglichkeit des Vergleichs von Enzymaktivitäten in verschiedenen Lebensaltern auch bei Vorliegen pathologischer Veränderungen.

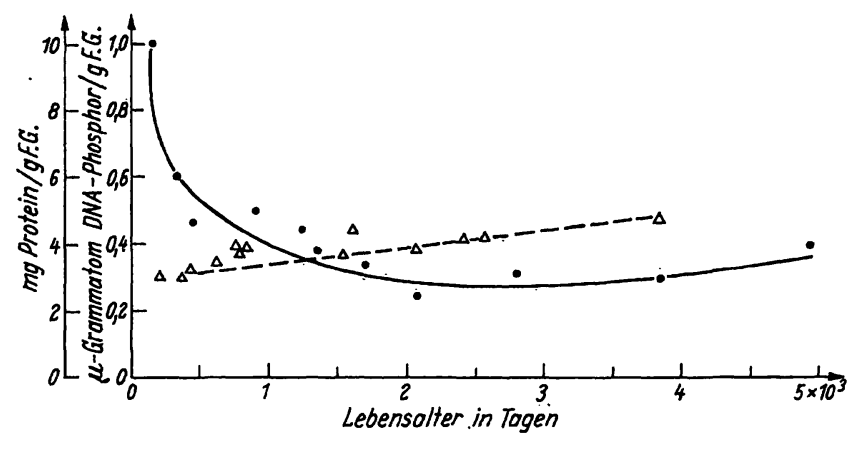

Abb.1

Gehalt an Desoxyribonucleinsäure im Rinderarteriengewebe und Proteingehalt im zellfreien Uberstand von Rinderarteriengewebshomogenaten in Abhängigkeit vom Lebensalter.

Rechte Ordinate: $\mu$ g-Atom DNA-Phosphor/g Frischgewicht (•-•) linke Ordinate: $\mathrm{mg}$ Protein im Homogenatüberstand/g Frischgewicht $(\Delta-\Delta)$

Die Bestimmung des DNA-Gehaltes der Aorta des Rindes an Gewebsproben verschiedenen Lebensalters, deren Ergebnis in Abbildung 1 dargestellt ist, läßt erkennen, daß der DNA-Gehalt des Arteriengewebes beim Rind nach der Geburt schon innerhalb des ersten Lebensjahres einen steilen Abfall von 1000 auf etwa $500 \mu \mathrm{g}$-Atom DNA-Phosphor/g Frischgewicht zeigt, während der weiteren Lebensjahre jedoch konstant bleibt oder nur getingfügige Schwankungen aufweist. Dies bedeutet, daß die Zellzahl/g Frischgewebe während der Wachstumsphase im Arteriengewebe auf die Hälfte absinkt, ohne daß Angaben darüber möglich sind, ob die Abnahme auf Kosten eines bestimmten Zelltyps (Muskelzellen, Fibroblasten) erfolgt.

Die aus Frischgewebe extrahierbare Proteinmenge, die als lösliches Protein im zellfreien Uberstand des Homogenates erhalten wird, weist dagegen bei der Geburt die niedrigsten Werte auf (30 mg/g Frischgewebe) und hat mit zunehmendem Lebensalter ansteigende Tendenz (Werte bis $40 \mathrm{mg} / \mathrm{g}$ Frischgewebe).

Bezüglich des Gehaltes an DNA bestehen zwischen dem Arteriengewebe des Rindes und des Menschen Parallelen. Sie zeigen sich darin, daß es auch beim Menschen zu einem Abfall des DNA-Gehaltes von $450 \mu \mathrm{g}$-Atom DNA-Phosphor (Neugeborene) auf etwa $150 \mu \mathrm{g}$-Atom DNA-Phosphor/g Frischgewebe zwischen dem 40. und 80. Lebensjahr kommt. Die in Abbildung 2 eingezeichneten Werte sind Mittelwierte aus 85 Bestimmungen (3-15 Bestimmungen je Altersklasse).

\section{Altersabhängige Veränderungen der Enzymaktivi-} täten im Arteriengewebe des Rindes

In Abbildungen 3 und 4 sind die Ergebnisse der Einzelbestimmungen der Enzymaktivitäten von Glycosamino-Glykanohydrolasen und Peptidhydrolasen dargestellt. Sie lassen erkennen, $\mathrm{da} B$ N-Acetyl- $\beta$-D-glucosaminidase (EC 3.2.1.30) und $\beta$ - $\mathrm{D}$-Glucuronidase (EC 3.2.1.31) keine gerichtete Tendenz zur Aktivitätsänderung mit zunehmendem Alter zeigen. Trotz Streuung der Einzelwerte, die besonders bei der NAcetyl- $\beta$-D-glucosaminidase auffällt, bewegen sich ihre Enzymaktivitätswerte in einem Bereich zwischen 600 


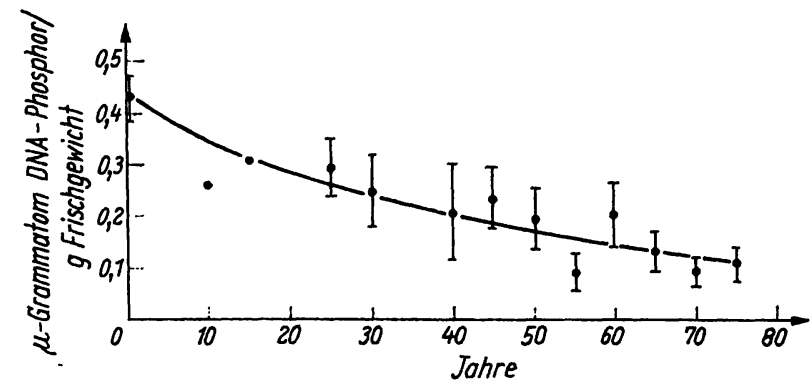

Abb. 2

Gehalt an Desoxyribonucleinsäure in menschlichem Arteriengewebe in Abhängigkeit vom Lebensalter. Die eingezeichneten Werte sind Mittelwerte aus 3-15 Bestimmungen je Altersklasse, für die, soweit berechenbar, die Standardabweichung eingezeichnet ist.

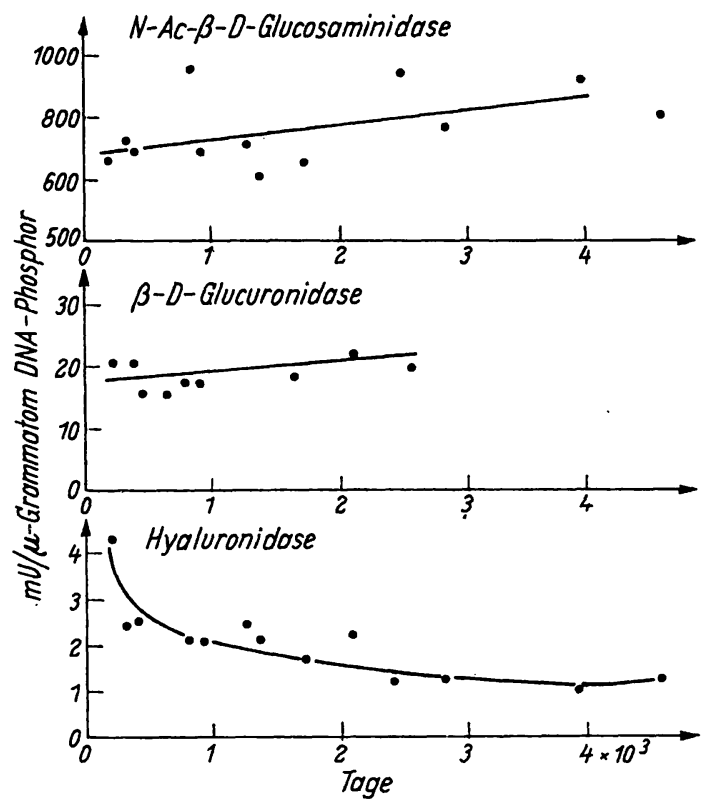

Abb. 3

Aktivität der $\mathrm{N}$-Acetyl- $\beta$-D-glucosaminidase, $\beta$-D-Glucuronidase und Hyaluronidase im Rinderarteriengewebe in Abhängigkeit vom Lebensalter.

und $800 \mathrm{mU}$. Die entsprechenden Werte für die $\beta$ Glucuronidase, die ebenfalls stärkere, altersabhängige Veränderungen vermissen lassen, liegen etwa $30 \mathrm{mal}$ niedriger. Bei der Beurteilung ist jedoch zu berücksichtigen, $\mathrm{da} \beta$ die Aktivitätswerte sich auf synthetische Substrate beziehen und $\beta$-Glucuronidase und N-Acetyl$\beta$-D-glucosaminidase gegenüber dem physiologischen Substrat (Chondroitin-4-sulfat-oligosaccharid) gleiche Umsatzraten zeigen (1).

Für die Aktivitätsbestimmung der Hyaluronidase (EC 3.2.1.35) mưßte aus den Enzympräparaten zuvor die $\beta$-D-Glucuronidase säulenchromatographisch abgetrennt werden, da deren Anwesenheit zusammen mit der N-Acetyl- $\beta$-D-glucosaminidase höhere Hyaluronidaseaktivitäten durch hydrolytische Abspaltung freien $\mathrm{N}$-Acetyl-glucosamins aus den Reaktionsprodukten der Hyaluronidasereaktion' vortäuscht. Beide Fremdaktivitäten wurden nach Ammoniumsulfatfällung 15-25 (Gew.\%) durch Gelfiltration abgetrennt und die Summe der in einem Aktivitätsgipfel enthaltenen Enzymaktivität auf die :eingesetzte Menge an Frischgewebe bzw. dessen DNA-Gehalt bezogen. Die Werte der Abbildung 3 entsprechen somit der „wahren Hyaluronidase". Die Altersverteilung der Hyaluronidaseaktivität unterscheidet sich von den beiden anderen Glucosidasen und von den Peptidhydrolasen (s. unten) dadurch, daß die Werte im Laufe des Lebens um mehr als $50 \%$ abfallen. Bemerkenswert ist auch, daß die Absolutaktivität der Hyaluronidase $10 \mathrm{mal}$ geringer ist als diejenige der beiden anderen Glykosidasen.

Im Gegensatz zu diesen Ergebnissen sind Kathepsin D und saure Carboxypeptidase der Rinderaorta durch einen Aktivitätsanstieg mit zunehmendem Alter gekennzeichnet. Die einschränkende Tatsache, daß es sich um Einzelbestimmungen handelt, wird durch die nur geringe Schwankung in den ersten Lebensjahren und den kontinuierlichen Aktivitätsanstieg bis zum 7. bzw. 13. Lebensjahr (2,6 bzw. $4,7 \times 10^{3}$ Tage) ausgeglichen.

3. Veränderungen der Enzymaktivitäten in Abhängigkeit vom Alter und Arteriosklerose im menschlichen Arteriengewebe

An menschlichen Arterien lassen sich altersbedingte und arteriosklerosespezifische Aktivitätsveränderungen nicht sicher voneinander abgrenzen, da zunehmendes Lebensalter auch von einem zunehmenden Schweregrad der Arteriosklerose begleitet ist und eine makroskopische Beurteilung und Aufteilung des Untersuchungsgutes keine hinreichende Basis für eine getrennte Analyse arteriosklerotischer und nicht arteriosklerotischer Gewebsabschnitte darstellt, da gerade in den makroskopisch nicht erkennbaren Frühstadien pathogenetisch wichtige Veränderungen zu erwarten sind.

Die in den Abbildungen 5 und 6 zusammengestellten Daten sind Mittelwerte von je 5-14 Doppelbestimmungen der in den verschiedenen Altersklassen durchgeführten Messungen. Einen Überblick über die Ergebnisse der statistischen Auswertung gibt Tabelle 1. In den höheren Altersklassen wurden Gewebsproben mit erkennbar stätkeren arteriosklerotischen Veränderungen und solchen mit geringerem oder kaum sichtbarem arteriosklerotischen Befall getrennt untersucht. Die Aktivitätskurven für N-Acetyl- $\beta$-D-glucosaminidase und $\beta$-D-Glucuronidase zeigen einen charakteristischen Verlauf: Während innerhalb der ersten drei Lebensdekaden keine wesentliche Aktivitätsänderung gemessen wird, kommt es im höheren Lebensalter zu einer kontinuierlichen Zunahme der Aktivität, die bei einer Gesamtaktivitätszunahme von über $100 \%$ zwischen dem 60. und 80. Lebensjahr Maximalwerte erreicht. Die Differenzen $z$ wischen der Altersklasse der Neugeborenen (bzw. zwischen 21. und 30. Lebensjahr) und dem 60. bis 80. Lebensjahr lassen sich statistisch sichern. Die Wahrscheinlichkeit $\mathrm{p}$ keiner Differenz der Mittelwerte ist für die $\mathrm{N}$-Acetyl- $\beta$-D-hexosaminidase $<0,001$, für die $\beta$-D-Glucuronidase $<0,001$. 


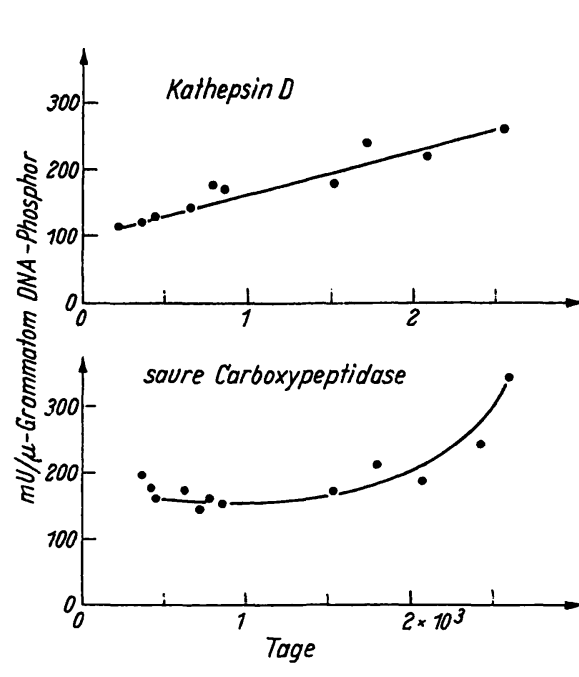

Abb. 4

Aktivität des Kathepsin $D$ und der sauren Carboxypeptidase in Abhängigkeit rom Lebensalter

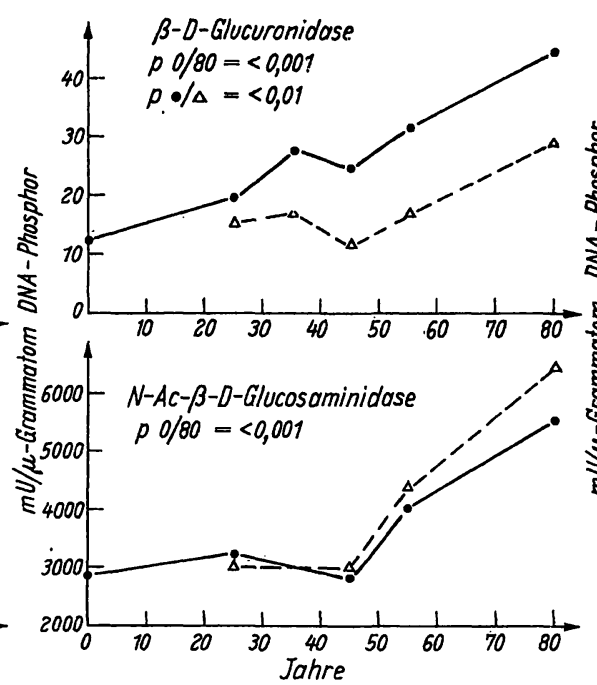

Abb. 5

Aktivität $\operatorname{der} \beta$-D-Glucuronidase und NAcetyl- $\beta$-D-glucosaminidase in menschlichem Arteriengewebe in Abhängigkeit vom Lebensalter und von Arteriosklerose. Mittelwerte aus 6 bzw. 5 Altersklassen mit je 6-14 Einzelbestimmungen

-- : Gewebsproben ohne makroskopisch sichtbare bzw. mit geringen arteriosklerosichtbare bzw. mit geringen arterio
tischen Veränderungen $\Delta-\Delta:$ Gewebsproben mit stärkeren arte-

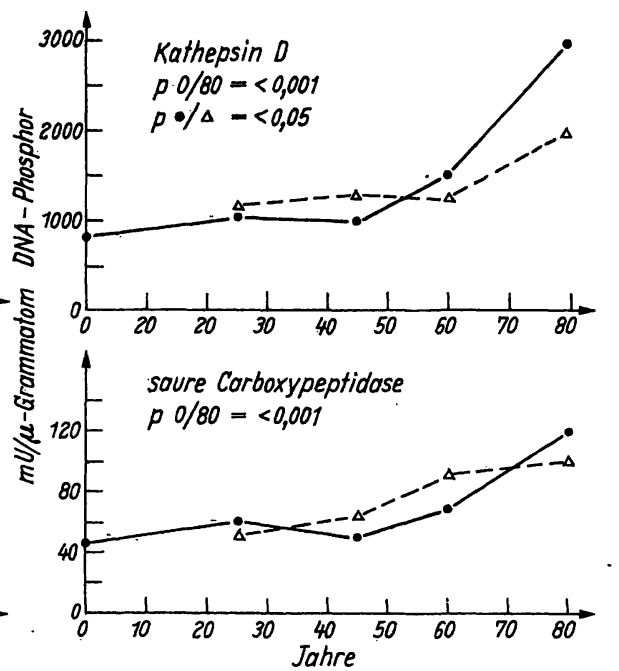

Abb. 6

Aktivität des Kathepsin D und der sauren Carboxypeptidase im menschlichen Arteriengewebe in Abhängigkeit vom Lebensalter und Arteriosklerose. Mittelwerte aus 5 Altersklassen mit je 6 bis 14 Einzelbestimmungen. Übrige Angaben wie in Abb. 5

Tab. 1

Wahrscheinlichkeit einer Differenz der Enzymaktivität menschlichen Arteriengewebes (Aorten) in verschiedenen Altersklassen und bei unterschiedlichem Arteriosklerosegrad (AS). Alle angegebenen Werte sind mit dem Präfix < zu versehen

\begin{tabular}{|c|c|c|c|c|c|}
\hline \multirow[b]{2}{*}{ Enzym } & \multicolumn{5}{|c|}{ Wahrscheinlichkeit p einer nicht signifikanten Differenz der Untersuchungsgruppen } \\
\hline & $\begin{array}{l}\text { Neugeb. I } \\
61-80 \mathrm{~J} .\end{array}$ & $\begin{array}{c}21-40 \mathrm{~J} .1 \\
61-80 \mathrm{~J} .\end{array}$ & $\begin{array}{r}41-50 \mathrm{~J} \cdot{ }^{\prime}{ }^{\prime} \\
\end{array}$ & $61-60 \mathrm{~J} \cdot 1$ & $\begin{array}{l}61-80 \mathrm{~J} \cdot \\
\text { (beginn. As)/ } \\
6.1-80 \mathrm{~J} . \\
\text { (stärkere AS) }\end{array}$ \\
\hline $\begin{array}{l}\beta \text {-D-N-Acetyl-glucosaminidase } \\
\beta \text {-D-Glucuronidase } \\
\text { Kathepsin D } \\
\text { saure Carboxypeptidase }\end{array}$ & $\begin{array}{l}0,001 \\
0,001 \\
0,001 \\
0,001\end{array}$ & $\begin{array}{l}0,15 \\
0,001 \\
0,001 \\
0,01\end{array}$ & $\begin{array}{l}0,001 \\
0,001 \\
=\end{array}$ & $\begin{array}{l}0,05 \\
0,001 \\
0,02 \\
0,02\end{array}$ & $\begin{array}{l}0,10 \\
0,01 \\
0,025 \\
0,8\end{array}$ \\
\hline
\end{tabular}

Differenzen zwischen Gewebsproben mit geringerem und stärkerem arteriosklerotischen Befall waren für die $\mathrm{N}$-Acetyl- $\beta$-D-glucosaminidase statistisch nicht $\mathrm{zu}$ sichern, für die $\beta$-D-Glucuronidase jedoch eindeutig. Bei nur geringgradigem arteriosklerotischen Befall ist die $\beta$-D-Glucuronidase-Aktivität um fast $30 \%$ höher als in Gewebsabschnitten mit stärkeren arteriosklerotischen Veränderungen und teilweise bindegewebigen Sekundärreaktionen.

Dieser Unterschied entspricht einer statistischen Wahrscheinlichkeit von $\mathrm{p}<0,01$. Bemerkenswert ist, $\mathrm{da} B$ beim Menschen die Absolutwerte für $\mathrm{N}$-Acetyl- $\beta$-Dglucosaminidase etwa $5 \mathrm{mal}$ höher liegen als beim Rind, für die $\beta$-D-Glucuronidase jedoch geringfügig niedriger. Die Hyaluronidase zeigt beim Menschen eine deutlich geringere Aktivität, die beim Neugeborenen noch erfaßbar, in den höheren Altersklassen unter den gewählten Aufarbeitungsbedingungen jedoch unter der Nachweisgrenze lag.

Eine Zunahme der Enzymaktivität in Abhängigkeit vom Lebensalter ist auch bei den Peptidhydrolasen erkennbar. Nach einer Periode konstanter Aktivität in den ersten Lebensjahrzehnten kommt es im Durchschnitt zu einem Aktivitätsanstieg um 200 bis 300\% (Einzelwerte bis 500\%), der statistisch mit einer Wahtscheinlichkeit $\mathrm{p}$ für die Protease von $<0,001$ und für die Carboxypeptidase mit $<0,001$ gesichert ist. Darüber hinaus läßt sich für die Protease für Gewebsproben verschiedener arteriosklerotischer Schweregrade eine statistisch signifikante Differenz nachweisen $(p<0,025)$ mit hohen Enzymaktivitäten für geringeren und um fast $100 \%$ niedrigeren Aktivitäten für fortgeschrittenen arteriosklerotischen Befall. Die Absolutaktivitäten liegen (bezogen auf den Zellgehalt) bei Mensch und Rind in gleicher Größenordnung.

\section{Diskussion}

Bei der Beurteilung von Enzymaktivitätsänderungen in der Arterienwand in Abhängigkeit vom Lebensalter ist zu berücksichtigen, $\mathrm{da} B$ die Bezugsbasis für die $\mathrm{Ak}$ tivitätsangabe das Profil der Alterskurve entscheidend bestimmt. Bezieht man die Enzymaktivität auf $\mathrm{mg}$ Extraktprotein, so ist bei allen untersuchten Enzymen . während des ersten Lebensjahres beim Rind und während 
des ersten Dezenniums beim Menschen ein eindeutiger Aktivitätsabfall nachweisbar. Setzt man jedoch die gemessenen Enzymaktivitäten zur Zahl der Zellen des Gewebes, d. h. zu der gleichzeitig ermittelten Konzentration an DNA in Beziehung, so bleiben die Enzymaktivitäten während dieses Zeitraumes in allen Fällen nahezu konstant. Für den weiteren Verlauf der Enzymaktivitäts-Alterskurve ist die Tatsache maßgeblich, daß der DNA-Gehalt des Gewebes sich im Laufe des Lebens entweder weiter vermindert oder innerhalb einer gewissen Schwankungsbreite konstant bleibt. Die Abnahme des DNA-Gehaltes ist zum mindesten beim Menschen relativ, da auch das Frischgewicht der Aorta beim erwachsenen Menschen im Laufe des Lebens von etwa $25 \mathrm{~g}$ auf $50-100 \mathrm{~g}$ ansteigt (7), so daß sich das von der Einzelzelle zu versorgende Areal etwa verdreifachen muß.

Bei den an Rinderaorten erhaltenen Ergebnissen bezüglich der Änderung von Enzymaktivitäten in Abhängigkeit vom Lebensalter fällt auf, daß die auf den DNA-Gehalt bezogenen Enzymaktivitäten sowohl zunehmen (Kathepsin D, saure Carboxypeptidase) als auch abnehmen (Hyaluronidase) können, obwohl nachgewiesen ist, daß alle hier untersuchten Enzyme lysosomalen Ursprungs sind (8). Daraus ist zu folgern, daß Kathepsin D und Hyaluronidase entweder nicht in den gleichen Zelltypen, oder innerhalb der Einzelzelle in verschiedenen Lysosomentypen gebildet werden und diese wiederum unterschiedlichen Altersveränderungen unterliegen.

Die Tatsache, daß ferner ein Anstieg der Enzymaktivität trotz Abnahme des DNA-Gehaltes gemessen werden kann, läßt den Schluß zu, daß entweder die Stoffwechselaktivität der Einzelzelle des Arteriengewebes im Alter erhöht ist, oder aber die im Arteriengewebe vorhandenen Zelltypen ein unterschiedliches Enzymverteilungsmuster aufweisen und die Abnahme der Zellzahl vorwiegend Zellen mit nur geringer Aktivität der Enzyme des Chondroitinsulfat-Proteinabbaues betrifft. Gegen diese Vorstellung spricht allerdings die Tatsache, daß die hier untersuchten Enzyme in der Tunica intima (Fibroblasten, Fibrozyten) und Tunica media (ausschließlich Muskelzellen) (9) in gleicher Aktivität vorhanden sind, obgleich beide Arterienwandschichten aus ganz verschiedenen Zelltypen bestehen. Dem entspricht, daß Glucuronidaseaktivität histochemisch sowohl in Fibroblasten als auch in den Muskelzellen der Media nachgewiesen wurde (10). Analogieschlüsse aus den Verhältnissen in der Skelettmuskulatur sind allerdings nicht zulässig, da die Proteaseaktivität der Skelettmuskulatur höher, diejenige der $\beta$-D-Glucuronidase jedoch niedriger liegt als im Arteriengewebe (11). Im Hinblick auf den hier nachgewiesenen Anstieg beider Enzymaktivitäten im höheren Lebensalter bzw. bei Arteriosklerose ist diese Tatsache schwer vereinbar mit der Auffassung, daß Änderungen im Enzymverteilungsmuster durch Änderungen im prozentualen Anteil der einzelnen Zelltypen bedingt sind.
Die an der Rinderaorta gewonnenen Ergebnisse sind im Hinblick auf vergleichende Untersuchungen an menschlichen Arterien von Interesse. Da beim Rind mit zunehmendem Lebensalter zwar ebenfalls morphologische Veränderungen mit Intima-Veränderungen und Aufsplitterungen der Elastica interna beobachtet, der menschlichen Arteriosklerose analoge Veränderungen jedoch vermißt werden, gibt das Enzymmuster der Rinderarterien einen Hinweis auf physiologische Altersveränderungen. Im Gegensatz $z u$ den Altersveränderungen beim Rind kommt es beim Menschen jedoch zwischen dem 3. und 8. Lebensjahrzehnt $z u$ einem statistisch gesicherten Anstieg aller untersuchten Enzyme, und zwar in den Frühstadien der Arteriosklerose stärker, als bei fortgeschrittener Arteriosklerose. Untersuchungen über Aktivitätszunahmen von Protease (12), $\beta$-D-Glucuronidase $(13,14)$ und N-Acetyl- $\beta$ D-hexosaminidase (14) werden damit zwar ergänzt, jedoch insofern revidiert, als unsere Ergebnisse die Annahme einer höheren Aktivität von $N$-Acetyl- $\beta$-Dglucosaminidase und $\beta$-D-Glucuronidase bei Gewebsproben mit fortgeschrittener Arteriosklerose gegenüber Proben mit geringerem arteriosklerotischen Befall nicht bestätigen können. Auch zeigt die von uns beobachtete Altersveränderung $\operatorname{der} \beta$-D-Glucuronidaseaktivität in menschlichen Arterien einen anderen Verlauf als bei 1. c. (13) angegeben. Angaben über Veränderungen der Hyaluronidaseaktivität in Gewebshomogenaten (15) in Abhängigkeit vom Lebensalter besitzen ohnehin nur begrenzten Aussagewert, da es bei dem gleichzeitigen Anstieg der $\beta$-D-Glucuronidase und N-Acetyl- $\beta$-Dglucuronidase-Aktivität immer zu einer unspezifischen (methodisch bedingten) Erhöhung der gemessenen Hyaluronidaseaktivität kommt.

Die beobachtete Enzymaktivitätssteigerung bei der Spontanarteriosklerose des Menschen muß nicht Ausdruck einer katabolen Stoffwechsellage des Arteriengewebes sein, denn bei lysosomalen Enzymen ist für die Beurteilung ihres Stoffwechseleffektes nicht die gemessene Gesamtaktivität entscheidend, sondern jener unbekannte Anteil des Enzyms, der zum Zeitpunkt der Bestimmung in Funktion war (,effektive Enzymkonzentration"). Nach Berechnungen, denen die Daten für den tatsächlichen Stoffumsatz (biologische Halbwertszeit) und für die gesamte verfügbare Enzymaktivität zugrunde liegen (1), bettägt der stoffwechselaktive Anteil der lysosomalen Enzyme nur wenige Prozent der in den Lysomen als Reserve vorhandenen Enzymmenge. Beobachtungen, nach denen auch die Biosynthese der sulfathaltigen Polysaccharide in arteriosklerotischen menschlichen Arterien gesteigert ist (16), könnten allerdings darauf hinweisen, daß auch die Zunahme der Aktivität Chondroitinsulfat-Protein abbauender Enzyme Ausdruck eines erhöhten Stoffumsatzes der sauren Mucopolysaccharide in den Frühstadien der Arteriosklerose ist.

Die Deutsche Forschungsgemeinschaft und der Fonds der Chemischen Industric haben die Arbeit durch Sachbeihilfen in dankenswerter Weise unterstützt. 


\title{
Literatur
}

1. Held, E., O. Hoefrele, G. Reich, U. Stein, E. Werizies und E. BUDDECKE, diese Z., vorstehend. - 2. KAPLAN, D. und $K$. MeYer, Proc. Soc. exper. Biol. Med. N.Y. 105, 78 (1960). BudDECKE, E. J. Atheroscler. Res. 2, 32 (1962). - 3. Hauss, W. H., Verh. Dtsch. Ges. inn. Med., 69, 554 (1963). Hauss, W. H. und G. JUNGE-HüLsing, Dtsch. med. Wschr. 86, 763 (1961). - 4. BuDDecke E. und D. Platt, diese Z., 3, 4 (1965). - 5. Burton, K., Biochem. J. 62, 315 (1956). - 6. Kaltwasser, F., P. Wolters und J. PIEper, Clin. chim. Acta (Amsterdam) 15, 347 (1967). 7. MEYer, W. W., Virchows Arch. path. Anat. 320, 67 (1951). 8. Filipovic, I. und E. BudDECKE, Hoppe-Seylers Z. physiol. Chem. im Druck. - 9. AdAMrs, C. W. M., Vascular Histochemistry, S. 33.
LLOYX-LUKe, Ltd., London (1967). - 10. ZEMPLÉNYX, T., Z. LojDA u. O. Mrhová, Atherosclerosis and its Origin, S. 504, ed. by M. Sandler u. G. H. Bourne, Academic Press., New York (1963). '11. Reich, G., Dissertation (Math.-Naturwiss. Fakultät, Univ. Tübingen) (1966). Hozfere, O., Dissertation (Math.-Naturwiss. Fakultät, Univ. Tübingen) (1966). -12. KIRK, J. E., J. Gerontol. 17, 158 (1962). - 13. BRANwORd, A. W. und C. J. CARR, Lancet II, 1254 (1960). - 14. DYrraye M. und J. E. KIRK, J. Gerontol. 11, 33 (1956). - 15. Platit, D., Klin. Wschr. 45, 92 (1967). 16. Haușs, H. W., G. Junge-Hülsing, K. J. Matthes und W. WrRTH, J. Atheroscler. Res. 5, 451 (1965).
Prof. Dr. E. Buddecke 44 Münster (Westf.) Waldeyerstraße 15

\section{Eine automatisierte Bestimmung von anorganischem Phosphat}

\author{
Von G. F. Hoppe-SEYLER und G. GundLACH \\ Aus der Klinisch-Chemischen Abteilung (Leiter: Priv.-Doz. Dr. G. Gundlach) der Urologischen Üniversitätsklinile \\ Homburg/Saar (Direktor: Prof. Dr. C. E. Alken)
}

(Eingegangen am 13. Dezember 1967)

\section{Herrn Prof. Dr. Dr. Ernst Schütte qum 60. Geburtstag qugeeignet}

Eine einfache, empfindliche, automatisierte Methode zur Bestimmung des anorganischen Phosphates in Serum, Harn und biologischen Flüssigkeiten wird beschrieben. Der Molybdänphosphorsäurekomplex wird durch Hydrazinsulfat bei $95^{\circ}$ reduziert.

Eine Modifikation der angegebenen Technik erlaubt die automatische Bestimmung sehr gẹtinger P-Konzentrationen $(0,05$ ppm P).

Die Interferenz stabiler Phosphatester und anderer Substanzen wird diskutiert.

A simple, sensitive, automated method is described for the determination of inorganic phosphate in serum, urine, and other biological fluids. The molybdenum-phosphoric acid complex is reduced by hydrazine sulphate at $95^{\circ}$.

The method can be modified for the automatic determination of very low phosphate concentrations $(0.05 \mathrm{ppm} . \mathrm{P})$. Interference by stable phosphate estets and other substances is discussed.

Die Grundlage der gebräuchlichen Phosphatbestimmungen ist die Reduktion der Molybdänphosphorsäure zu Molybdänblau. Empfindlichkeitsunterschiede der einzelnen Methoden beruhen auf der Verwendung verschiedener Reduktionsmittel. Für die automatische Bestimmung wird das Verfahren nach FISKE und SubBARow mit dem Reduktionsmittel 1, 2, 4-Aminonaphtholsulfosäure empfohlen $(1,2)$.

Voraussetzung für die Anwendung einer Methode in der klinisch-chemischen Routine und zur Durchführung von Reihenuntersuchungen ist die exakte Reproduzierbarkeit der Meßwerte und eine gute Haltbarkeit der Reagenzien. Dieser Forderung entspricht die Reaktion nach Bolz und Mellon (3), die Hydrazinsulfat als Reduktionsmittel verwendet, einen mindestens $12 \mathrm{Stdn}$. haltbaren Farbkomplex bildet und gegenüber dem vorgenannten Verfahren den Vorteil höherer Empfindlichkeit hat (4).

\section{Material und Methoden \\ Reagenzien}

Schwefelsäure p. a. (Merck 731)

Ammoniummolybdat (Merck 1182)

Hydrazinsulfat (Merck 4602)

Salzsäure p. a. (Merck 317)
Lösungen

A. Methode für Serum (10-100 ppm P)

1. $\mathrm{Zu} 4500 \mathrm{~m} l$ dest. Wasser werden $270 \mathrm{~m} l$ Schwefelsäure gegeben. Nach Mischen und Abkühlen ${ }^{1}$ ) der Lösung fügt man $30,0 \mathrm{~g}$ Ammoniummolybdat zu.: Auffüllen mit dest. Wasser auf $5000,0 \mathrm{ml}$.

2. 0,4 proz. Hydrazinsulfat

3. $1 \mathrm{~N} \mathrm{HCl}$

B. Mikromethode (0,05-1 ppm P)

1. $\mathrm{Zu} 4000 \mathrm{~m} l$ dest. Wasser werden $665 \mathrm{~m} l$ Schwefelsäure gegeben. Nach Mischen und Abkühlen ${ }^{1}$ ) der Lösung fügt man $100,0 \mathrm{~g}$ Ammoniummolybdat zu. Auffüllen mit dest. Wasser auf $5000,0 \mathrm{ml}$.

2. 1 proz. Hydrazinsulfat

3. 0,18 proz. $\mathrm{HCl}$

\section{Ausfübrung}

A. Methode für Serum

Das Verhältnis der Reagenzmengen zueinander geht aus Abbildung 1a hervor, wobei die Zablenangaben an den Pumpschläuchen den geförderten $\mathrm{m} / / \mathrm{Min}$. entsprechen. Reagenzkonzentrationen s. Lösúngen.

1) Es ist unbedingt erforderlich zuerst die Schwefelsäurelösung herzustellen und vor Zugabe des Ammoniummolybdatemolybdates gut abkühlen zu lassen. Die Reagenzlösungen sind dann in jeder Konzentration beständig. 